\title{
Negative Space: Workspace Awareness in 3D Face-to-Face Remote Collaboration
}

\author{
Maurício Sousa \\ INESC-ID, IST, ULisboa \\ antonio.sousa@tecnico.ulisboa.pt \\ Daniel Simões Lopes \\ INESC-ID, IST, ULisboa \\ daniel.s.lopes@tecnico.ulisboa.pt
}

\author{
Daniel Mendes \\ INESC-ID, IST, ULisboa \\ danielmendes@tecnico.ulisboa.pt
}

\author{
Rafael K. dos Anjos \\ INESC-ID, IST, ULisboa \\ rafaelkuffner@tecnico.ulisboa.pt
}

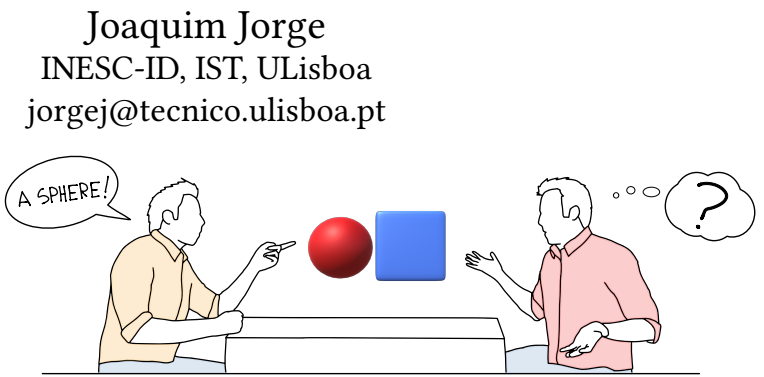

Figure 1: Example illustration depicting the occlusion issue present when people have opposing points-of-view.

and remote person should be always visible. Indeed, using a transparent display metaphor, two participants are able to see one another and share digital content, rendered between them, that can be jointly manipulated by both. Yet, in plain face-to-face interactions mediated by displays, people have no common orientation of right or left. Clearboard [Ishii and Kobayashi 1992] addresses this issue by mirror-reversing the remote person's video stream, producing gaze and pointing awareness, since $2 \mathrm{D}$ graphics and text can thus be corrected to the participant's point-of-view. This approach has been the subject of research for 2D content collaborative manipulation [Wood et al. 2016]. However, 3D digital content gives rise to detracting issues that affect and impair workspace awareness. Participants do not share the same forward-backwards orientation, occlusions can affect the understanding of where or what the remote person is pointing at. Also, contrary points-of-view can result in different perceptions or even serious communication missteps, as illustrated in Figure 1.

This work focuses on assessing workspace awareness using variations of the shared workspace settings, individual point-of-view and remote user's representation. For this purpose, we conducted an evaluation comparing task performance and user preferences under four different conditions. We employed an evaluation environment inspired by the "portal to a distant office" concept from Wen et al. [Wen et al. 2000] creating a virtual space between two real spaces. From the results, we conceptualize the Negative Space, an approach to face-to-face remote collaboration, creating a shared virtual workspace linking two physical remote spaces.

\section{EVALUATION}

We set out to assess if different manipulations of person and task spaces can enhance workspace awareness.We developed a full body telepresence prototype and implemented four different workspace conditions. For this, we designed a collaborative 3D assembly task where an Instructor guides a remote Assembler to reach the correct solution of a toy problem using cubes. Our goal was to study the

\author{
Permission to make digital or hard copies of part or all of this work for personal or \\ classroom use is granted without fee provided that copies are not made or distributed \\ for profit or commercial advantage and that copies bear this notice and the full citation \\ For all other uses, contact the owner/author(s). \\ VRCAI '19, November 14-16, 2019, Brisbane, QLD, Australia \\ (C) 2019 Copyright held by the owner/author(s) \\ ACM ISBN 978-1-4503-7002-8/19/11. \\ https://doi.org/10.1145/3359997.3365744 \\ When designing for face-to-face collaboration it is necessary to take \\ into account how to address interactions in a shared 3D workspace. \\ Ishii et al. [Ishii and Kobayashi 1992] suggested that both workspace
}


participants' point-of-view, remote participant's embodiment and workspace rendering. For point-of-view we considered that participants could observe workspace in usual opposing points-of-view or simulating an identical viewing experience. Also, similarly to Ishii et al. [Ishii et al. 1993], embodiment and workspace variables could both be horizontally inverted or not.

Our evaluation followed a within subjects design with four conditions: (1) Real Life Face-to-face (RL): Derived from the real world face-to-face scenario, both participants can see each other and the workspace as if they were in opposite ends. (2) Simulated Side-byside (SS): While remaining face-to-face in regard to the embodied representation, participants share the same point-of-view of the workspace, in a way that simulates a side-by-side approach. (3) Mirrored Person (MP): Participants share the same point-of-view, yet the instructor's embodied representation is horizontally inverted to match the reference space. (4) Mirrored Workspace (MW): With an identical point-of-view, participants also share faithful face-to-face embodiment representations of each other, although the assembler's workspace is horizontally inverted.

Results show an absence of significant differences in task performance and, for user preferences, statistical significant differences were found on instructors' answers. This happened because it was mostly the instructor who did the calculations regarding reference frames, which rendered all conditions alike to the assembler. Although participants established the informal shared protocol to calibrate reference frames and achieved similar performance in all conditions, a reflected workspace was clearly identified as being more difficult than an exact representation. We argue that the cognitive workload of being constantly converting coordinates between both frames is mentally demanding. In complex scenarios, where it is imperative for both participants to observe the same details, the RL condition is unfit. This and the cognitive cost associated to the MW condition, leads us to suggest that, for this kind of scenarios, having an exact workspace with an identical point-of-view is highly desirable. The choice between SS or MP will be dependent on whether the accuracy of the remote person's representation is more relevant than the consistency between the person and task spaces, respectively.

\section{NEGATIVE SPACE}

Previous research on remote face-to-face collaboration have successfully contributed full-body telepresence approaches with integrated person-task spacesYet, most focus on cooperative interactions with 2D content, although collaboration in design and review of $3 \mathrm{D}$ virtual models is crucial in several domains.

With this in mind, we introduce Negative Space as a conceptual platform with a set of rules for future works on remote collaboration. It is characterized as a virtual space that serves as a gateway between two physical rooms where collaborative 3D interactions can occur. From the evaluation results, we devised Negative Space as a medium to support discussions on shared views over the 3D content and, as such, it shall offer participants Identical points-ofview over Exact copies of the workspace. We also enforce the usage of real-time 3D reconstructions of remote people for improved perception. Our approach can be advantageous in avoiding communication breakdowns by making many gestures and deictic idioms easier to share and understand between participants.

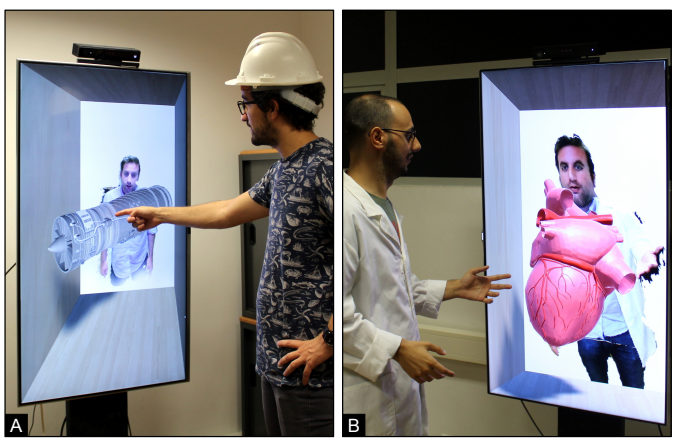

Figure 2: The Negative Space concept can be applied in multiple usage scenarios requiring visualization, design and review of virtual 3D models. Notable examples are A) engineering industries and the $B$ ) healthcare.

Similarly to Ishii et al. [Ishii and Kobayashi 1992], Negative Space exploits the benefits of a see-through display. By positioning the virtual content between two people, participants are able to profit from normal face-to-face interactions as if they were physically colocated. This contributes to the overall workspace and situational awareness, since participants are able to observe the other person's gaze direction, deictic gestures and actions, while performing selection and manipulation tasks related to multiple occupational fields, such as engineering, industrial, architecture and medical, as demonstrated in Figure 2.

\section{CONCLUSIONS}

In this work we presented an evaluation of several combinations of different points-of-view, and workspace and embodiment characteristics to study remote face-to-face collaborative work on 3D shared content, with the objective of achieving a consistent and seamless reference space between participants while promoting workspace awareness. As a consequence of the results' analysis, we conceptualize Negative Space, a telepresence approach that enables full-body face-to-face communication and creates a virtual task space between two remote spaces, where interactions with 3D objects can occur.

\section{ACKNOWLEDGMENTS}

This work was supported by FCT through grants UID/CEC/50021/ 2013 and IT-MEDEX PTDC/EEISII/6038/2014.

\section{REFERENCES}

Hiroshi Ishii and Minoru Kobayashi. 1992. ClearBoard: A Seamless Medium for Shared Drawing and Conversation with Eye Contact. In Proceedings of the SIGCHI Conference on Human Factors in Computing Systems (CHI '92). ACM, New York, NY, USA, 525-532. https://doi.org/10.1145/142750.142977

Hiroshi Ishii, Minoru Kobayashi, and Jonathan Grudin. 1993. Integration of interpersonal space and shared workspace: ClearBoard design and experiments. ACM Transactions on Information Systems (TOIS) 11, 4 (1993), 349-375.

Wei-Chao Wen, H. Towles, L. Nyland, G. Welch, and H. Fuchs. 2000. Toward a compelling sensation of telepresence: demonstrating a portal to a distant (static) office. In Proceedings Visualization 2000. VIS 2000 (Cat. No.00CH37145). 327-333. https://doi.org/10.1109/VISUAL.2000.885712

Erroll Wood, Jonathan Taylor, John Fogarty, Andrew Fitzgibbon, and Jamie Shotton. 2016. ShadowHands: High-Fidelity Remote Hand Gesture Visualization Using a Hand Tracker. In Proceedings of the 2016 ACM on Interactive Surfaces and Spaces (ISS '16). ACM, New York, NY, USA, 77-84. https://doi.org/10.1145/2992154.2992169 